of bulbar involvement came on. Death four months later. No P.M. Probably a tuberculous process commencing in the mid brain ; $v$. Lees, Vol. III; Taylor, Vol. XVIII; Batten, Vol. XXX.

Vol. XXX.

Batten, p. 116. "Bilateral ophthalmoplegia externa in an infant." A case resembling Jessop's, vide supra. The child showed also weakness of right arm and leg, with constant rhythmic movements, and subsequent convulsions on right side. Death after 6 months. P.M., a large tuberculous mass involving optic thalamus and corpora quadrigemina with a second tuberculous mass on the surface of left occipital cortex.

VoL. XXXII.

McMullen, p. 110. " External ophthalmoplegia of long standing." Commenced at age of 8. Pupils and accommodation normal.

Bradburne, p. 142. "Hereditary ophthalmoplegia in five generations." Sixteen out of thirty-seven individuals affected in five generations. In most of the cases all the external muscles were affected and ptosis was present in some but not all the cases. In the few cases where it is noted, pupillary movements and accommodation seem to have escaped.

Vol. XXXIII.

Cunningham, p. 156. "Paresis of both internal recti." Probably belongs to the class of case where a lesion in the posterior longitudinal bundle interferes with lateral deviation. Movements on convergence were not so markedly affected.

VoL. XXXIV.

Coats, p. 262. "Congenital partial paralysis of the oculo-motor nerve with cyclical contraction and dilatation of the pupil." A case in which rhythmical action of the pupil of the left eye accompanied a partial paralysis of branches of the left third. Partial ptosis. Complete loss of upward movement. Inward and downward movements defective.

VoL. XXXVIII.

James Taylor, p. 168. " Paralysis of ocular muscles." Occurring in : 1, myasthenia gravis : 2, Grave's disease ; 3, lethargic encephalitis.

F. E. Batten, p. 172. "Cases of lethargic encephalitis." These were some of the earliest cases of lethargic encephalitis shown in this country. Vol. XL.

Cross, p. 277. "The nerve paths and centres concerned in sight." A very tull account of the anatomical arrangement of the nerve centres and tracts including the comparative anatomy.

\title{
ANNOTATIONS
}

\section{Swanley Ophthalmia School and Non-Pauper Children}

It has often been said that the British Constitution grew and was not made; this statement might also be well applied to the arrangements for dealing with infectious and contagious diseases in the Metropolis.

The Metropolitan Asylums Board was established in 1867, by an order of what was then called the Poor Law Board, for the reception and relief of poor persons chargeable to some union or parish of London, infected with fever or smallpox, or suffering from insanity. The Poor Law Board subsequently became the Local Government Board, and in 1883 the Disease Prevention Act (London) removed the civil disabilities which had till then been attached to admission into the Metropolitan Asylums Boards Hospitals. 
The Poor Law Act, 1889, empowered the Board to admit nonpauper cases of fever, diphtheria, and smallpox.

In 1911 the Local Government Board sanctioned the admission to any of the infectious hospitals of poor persons suffering from such infectious or contagious disease other than those already mentioned, as they might thereafter determine. In accordance with this order they in 1912 sanctioned the admission of children suffering from measles and whooping cough, and later in the same year, subject to certain restrictions, the admission of non-pauper patients suffering from those diseases.

By an order issued in 1897 the Local Government Board constituted the Metropolitan Asylums Board the central authority in the Metropolis for dealing with various classes of Poor Law children, amongst them those suffering from ophthalmia. As the outcome of this order, the Metropolitan Asylums Board in 1903-4 opened two schools, one at Swanley and the other at Brentwood, for the isolation, treatment, and education of Poor Law children suffering from contagious eye diseases. These schools provided accommodation for about 600 children and up to 1910 it proved barely sufficient for those sent to them for treatment. Since that date there has been a gradual and steady decline in the number of children affected with contagious eye diseases in the London Poor Law schools, due mainly to the isolation which the ophthalmia schools provided checking the spread of those diseases. As the result of this decrease it became possible to give up the Brentwood School as an ophthalmia school, and for some time now the Swanley School has only been partly occupied.

The only children eligible for admission to these ophthalmic institutions were those whose parents were in receipt of Poor Law relief. The children of parents who were more thrifty and who struggled against the acceptance of anything implying the taint of pauperism, if they contracted ophthalmia, became excluded from attendance at their day schools, had to attend frequently at hospitals for treatment, and owing to the absence of proper home attention and suitable hygienic surroundings, often dragged on for months and months a miserable existence.

In the summer of last year the Council of British Ophthalmologists, impressed by the number of children in London suffering from contagious eye disease. They drew up a report advocating the admission of non-pauper children suffering from contagious eye diseases into the Metropolitan Asylums Board Ophthatmia School. This they forwarded to the Minister of Health asking him to receive a deputation in order that they might lay the views of the Council more fully before him.

This request met with a cordial reception and in due course a deputation was received by Sir George Newman, who, after the 
members of the Council had presented their case, expressed himself as in complete sympathy with the object it had in view, and promised to do what he could to get non-pauper patients made eligible for admission to the Metropolitan Asylums Board's ophthalmia institution as had been done for admission to their fever hospitals.

The necessary negotiations and arrangements have been carried out, and now any child attending at a London County Council school, suffering from a contagious eye disease, can be admitted to the ophthalmia school at Swanley, provided accommodation for the class of children for which it was originally intended is not encroached upon.

The Council of British Ophthalmologists may be congratulated upon the success of their intervention in this matter, calculated as it is, not only to restore numerous poor children to health and efficiency, but also to be a benefit to the community by checking the spread of contagious maladies of the eye amongst its members.

\section{The Preparation of Scientific Papers}

Those who aspire to write for the medical press have been placed under a debt of gratitude by the Medical Research Council, the publications department of which has recently (January, 1921) issued a brochure entitled "Notes upon the Preparation of Monographs and Reports for Publication by the Council." It embodies much information likely to be useful to such writers, and not easy to come at by the usual channels. The primary object of the pamphlet appears to be to keep the cost of publications and reports down to the lowest possible figure, a precaution rendered the more necessary on account of the rapidly increasing cost of printing. Care should be taken that copy be typewritten, or, when that is not possible, the utmost care should be taken that proper names and titles should be written legibly. In any case, one side only of the paper should be used, and the MS. should be sent packed flat and not rolled or folded. An author should correct his typewritten copy before it leaves his hands. Reference lists, tables, etc., should be sent in on separate sheets of paper, which, so far as may be, should be of uniform size. When quotations are inserted in the text, it is suggested that, if more than four lines long, they should be marked by a vertical line placed on the left-hand side to indicate that they are to be printed in a separate type. New paragraphs should be indicated by indenting the first line of the new paragraph about two inches. Footnotes, as a rule, are not desirable. The best method of keeping and arranging references is by the so-called "Harvard System." (1) According to this plan, all works cited are collected together at the end of an article where they are arranged in alpha- 